\title{
Maxwell rigidity and topological constraints in amorphous phase-change networks
}

\author{
M. Micoulaut ${ }^{1}$, C. Otjacques ${ }^{2}$, J.-Y. Raty ${ }^{2}$, C. Bichara $^{3}$ \\ ${ }^{1}$ Laboratoire de Physique Théorique de la Matière Condensée, \\ Université Pierre et Marie Curie, Boite 121, CNRS UMR 7600, 4 place Jussieu, 75252 Paris Cedex 05, France \\ ${ }^{2}$ Physique de la Matière Condensée, B5, Université de Liège, B4000 Sart-Tilman, Belgium \\ ${ }^{3}$ Centre Interdisciplinaire de Nanoscience de Marseille (CINaM ), CNRS and Aix-Marseille Universities, Campus \\ de Luminy, Case 913,13288 Marseille, France
}

\begin{abstract}
By analyzing first-principles molecular-dynamics simulations of different telluride amorphous networks, we develop a method for the enumeration of radial and angular topological constraints, and show that the phase diagram of the most popular system Ge-Sb-Te can be split into two compositional elastic phases: a tellurium rich flexible phase and a stressed rigid phase that contains most of the materials used in phase-change applications. This sound atomic scale insight should open new avenues for the understanding of phase-change materials and other complex amorphous materials from the viewpoint of rigidity
\end{abstract}

Keywords: Rigidity theory and rigidity transitions, network glasses, telluride phase change materials. PACS: $61.43 . F s$

\section{INTRODUCTION}

Glass networks have been found to display rather unusual and unique properties over a limited range of values of the mean coordination number $\langle r\rangle$, related to a alloying concentration $x$. Compelling evidence exists on the fact that networks with a mean coordination number of $\langle\mathrm{r}\rangle \sim 2.4$ display quite different properties from those at low $\langle\mathrm{r}\rangle \sim 2$ or high $<\mathrm{r}>\sim 3$ [1]. Along these lines, it has appeared that the glass forming tendency of melts is optimized when the network mean coordination numbers equals the "magic" number of $\langle\mathrm{r}\rangle=2.4$. Further developments of such mean field ideas have led to the identification of a floppy to rigid transition [2],[3] and to the suggestion that networks at $\langle r\rangle\langle 2.40$ are flexible (or mechanically deformable, or underconstrained, or hypostatic) whereas networks with $\langle r\rangle\rangle 2.40$ are stressed rigid (or overconstrained, or hyperstatic) [2]. At $\langle\mathrm{r}\rangle=2.4$, networks are isostatically rigid. This phenomenological framework (the Phillips-Thorpe mean-field rigidity transition) has dominated the physical understanding of the mechanical and thermal behaviour of network glasses for over nearly two decades [4].
An important breakthrough has been the discovery [5-7] of two elastic phase transitions (instead of one as initially thought) between the floppy and stressed rigid phases, defining a Boolchand intermediate phase (IP). This new picture has emerged from detailed Raman scattering results [5] and modulated differential scanning calorimetry (MDSC) on chalcogenide glasses [6,7]. Size increasing cluster approximations [8] on self-organized networks have revealed that these two transitions (rigidity, between the floppy phase and IP, and stress between the IP and the stressed-rigid phase) define a non-mean-field phase. Accordingly, the IP phase cannot be predicted on the basis of the mean field ideas, calling for a more fundamental approach to unravel its microscopic origin and rationalize experimental findings.

Since the location of the intermediate phase has been modeled with some success in sulphide and selenides, one may wonder to what extent this modeling can be applied for tellurides. One has to keep in mind, however, that the presence of the heavier tellurium having an increased propensity to form metallic-like bonds at the expense of covalent bonds makes the possible use of the so-called " $8-\mathrm{N}$ " (octet) rule problematic, $\mathrm{N}$ being the number of outer shell ( $\mathrm{s}$ and $\mathrm{p}$ ) electrons. A preliminary task is therefore to 
establish with confidence the mean-field counting for tellurides. This is the aim of the present contribution. We show that First Principles Molecular Dynamics can provide trajectories for various compositions of the Ge-Sb-Te system, and apply algorithms allowing for a neat estimate of bond-bending and bondstretching constraints. This leads ultimately to the creation of a rigidity map in the ternary telluride, the latter having important applications in the field of phase change memory devices as described next.

\section{PHASE CHANGE MATERIALS}

Phase change materials have received a huge attention in the very recent years, with various technological applications ranging from optical discs, such as CD-RW or DVD-RW discs to Phase-change memory (PRAM) devices, the latter being a promising candidate for future non-volatile computer memory [9]. In fact, prototype PRAM devices have demonstrated higher density and faster write times than flash memory. In both applications, the difference in electronic and optical properties between the crystalline and amorphous phase of the materials is at play. For optical storage, the crystalline phase is firstly hit by a nano-second light pulse from a high intensity "write" laser inside the drive which achieves a local (sub-micron) melting leading to amorphisation upon cooling, and change in reflectivity. A different pulse level may reverse the changes, erasing the recorded information by recrystallisation of the amorphous phase. A secondary "read" laser with less power will avoid melting and is used to measure the local reflectivity in order to detect if a bit is "on" (locally amorphous, low reflectivity) or "off" (locally crystalline, high reflectivity). PRAM uses the same principle but thermal induced changes between the crystalline and amorphous phases this time result from Joule heating though electrical current.

In Ge-Sb-Te alloys which are discussed here, three interesting compositional zones for phase change applications have been identified [10,11]: a pseudo-tie line with composition $\mathrm{GeTe}-\mathrm{Sb}_{2} \mathrm{Te}_{3}$, a tellurium deficient zone around $\mathrm{GeSb}_{6}$ and compositions close to $\mathrm{Sb}_{2} \mathrm{Te}_{3}$. Compositional changes lead to drastic changes in material properties thus in possibilities for PCM applications. For instance, increasing the germanium content along the $\mathrm{GeTe}-\mathrm{Sb}_{2} \mathrm{Te}_{3}$ join will lead to an increase of the melting point and the glass transition temperature favouring data retention, but to a decrease of the crystallization speed and the data transfer rate [10]. For reviews and details on the materials, we refer the reader to the excellent literature produced by $\mathrm{M}$. Wuttig and his co-workers [12].

\section{MAXWELL CONSTRAINT COUNTING}

Bond constraint counting, as initially introduced by Phillips and Thorpe, can be easily computed in random networks when only nearestneighbour forces dominate between atoms. Thorpe and co-workers [3] have furthermore demonstrated that networks constrained only by these local forces can be analyzed from the eigenmodes of the dynamical matrix (of dimensionality $3 \mathrm{~N}$ ) which represent the zerofrequency (floppy) modes. When these modes are present, the network can be deformed with a low cost in energy and is considered as flexible.
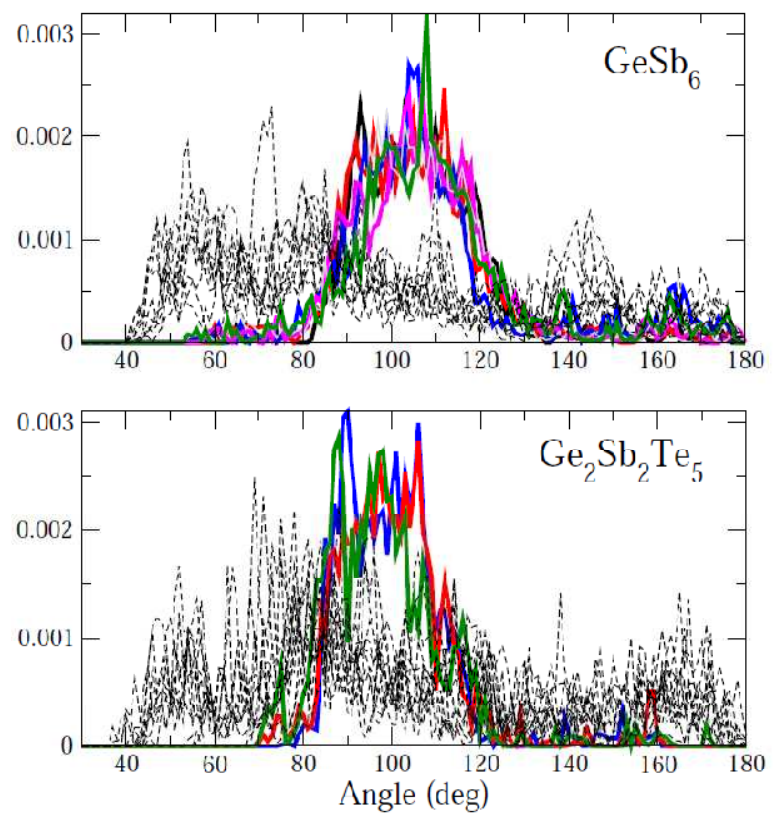

FIGURE 1. Having selected a maximum of $\mathrm{N}=6$ neighbors around a central germanium atom, on can compute $\mathrm{N}(\mathrm{N}$ 1)/2=15 partial bond angle distribution in $\mathrm{GeSb}_{6}$ and $\mathrm{Ge}_{2} \mathrm{Sb}_{2} \mathrm{Te}_{5}$ amorphous alloys which are all represented here. The colored curves correspond to distributions having a low standard deviation $\sigma_{\theta \mathrm{ij}}<20^{\circ}$ (see also Fig. 2), and which lead to intact angular constraints. One should also notice the centroid of the distributions, either located at the tetrahedral angle $109^{\circ}$ (for $\mathrm{GeSb}_{6}$ ), or somewhat lower than $100^{\circ}$, indicative of an octahedral site. See Ref. [14] for details.

For a network made of atoms with concentration $n_{r}$ and coordination $r$, the total number of constraints per atom is :

$$
n_{c}=\frac{\sum_{r \geq 2} n_{r}[r / 2+(2 r-3)]}{\sum_{r \geq 2} n_{r}}=\frac{\bar{r}}{2}+2 \bar{r}-3
$$


and $N=\sum_{r \geq 2} n_{r}$ represents the total number of atoms of the network, the fraction $f$ of floppy modes being given by $f=3-n_{c} . \bar{r}$ represents here the network mean coordination number. The vanishing of the number of floppy modes [3] defines a single transition between a flexible and a stressed rigid phase charcaterized by $f=0$.
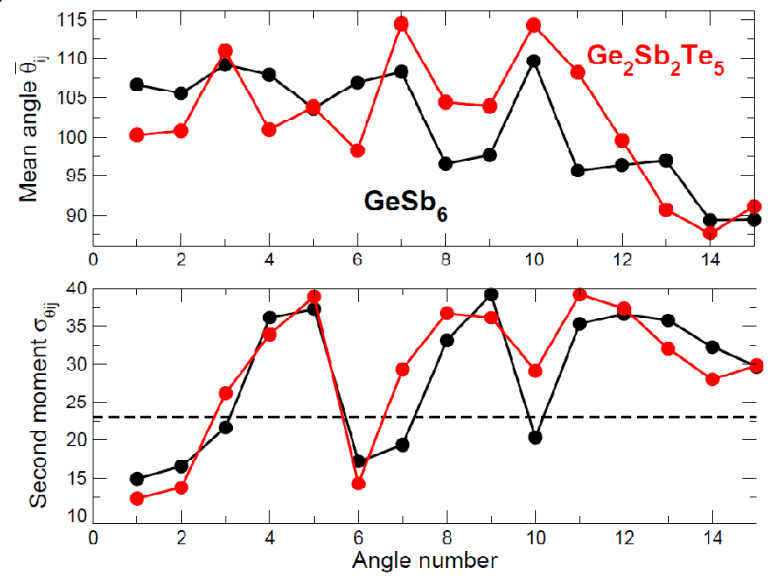

FIGURE 2. Mean angle and standard deviation of Gecentred partial angular distributions in two telluride PCMs. In the tetrahedral $\mathrm{GeSb}_{6}$, six constraints (black) are found (five independent, in harmony with direct Maxwell counting), whereas $\mathrm{Ge}_{2} \mathrm{Sb}_{2} \mathrm{Te}_{5}$ shows only three constraints (red).

As noticed from equ. (1), the main ingredient for the prediction of the number of constraints is the coordination number of the species involved. In sulphides and selenides, where such applications have been remarkably successful, these numbers can be easily obtained from the underlying local structure which is fully governed by the $8-\mathrm{N}$ (octet) rule yielding an obvious network topology for bonding constraints. The situation appears to be slightly different with the heavier and more metallic tellurium which leads to more complicated and non-obvious (e.g. distorted octahedral and tetrahedral) local structures, as highlighted both from experiments $[9,10]$ and simulations [13].

One has therefore to rely on a combined approach. Amorphous structures from First Principles Molecular Dynamics (FPMD) have been generated for seven compositions in the $\mathrm{Ge}-\mathrm{Sb}$-Te phase diagram (Fig. 3, for simulation details, see also [14]), and are used as input for radial and angular constraint counting algorithms of bond-stretching and bond-bending interactions. The latter are evaluated by computing the standard deviations of partial bond angle distributions (PBAD, Fig. 1) which focus on a given angle with respect to neighbour ranking. Details of the method and more results can be found in Ref. [14].

\section{RESULTS}

Let us only emphasize some the main results found for selected (seven) compositions in the Ge-Sb-Te phase diagram:

- In ternary compositions, the coordination number (CN) of Te is always larger than 2. However this increased $\mathrm{CN}$ gives only rise to one angular constraint. The $\mathrm{CN}$ gives immediately the number of BS constraints (CN/2, see equ. (1)).

- $\mathrm{Ge}$ and $\mathrm{Sb}$ are always four-fold coordinated. However, for germanium, one finds a mixture of tetrahedral ( $\mathrm{T}$, with a fraction $\eta$ of tetrahedra) and defect octahedral (DO) sites, corresponding to angles $109^{\circ}$, and $90^{\circ}$, respectively (see Fig. 2). For the $\mathrm{Sb}$ atoms, it is found three angular constraints.

- As seen from Fig. 2, the alloy $\left(\mathrm{GeSb}_{6}\right)$ is found to be a nearly Ge tetrahedral network as the mean angles for the distributionsn are found close to $109^{\circ}$. Thus $\eta=1$, and one has 5 independent angular constraints.

- Although Ge is always four-fold coordinated (but with two local environements, $\mathrm{T}$ or $\mathrm{DO}$ ), the number of angular constraints is given by $\mathrm{n}_{\mathrm{BB}}=3(1-\eta)+5 \eta=3+2 \eta$ (Fig. 2) For instance, in $\mathrm{Ge}_{2} \mathrm{Sb}_{2} \mathrm{Te}_{5}$, it is found from FPMD that $\eta=20 \%$, leading to $\mathrm{n}^{\mathrm{BB}}=3.4$.

Having these results in hand, i.e. coordination numbers and angular constraints, we can compute the total number of constraints per atom of $\mathrm{a} \mathrm{Ge}_{x} \mathrm{Sb}_{y} \mathrm{Te}_{1-x-y}$ system, equal to:

$$
\begin{aligned}
& n_{c}=n^{B S}+n^{B B}=\left[4(x+y)+\frac{r_{T e}}{2}(1-x-y)\right] \\
& +x(3+2 \eta)+3 y+1-x-y
\end{aligned}
$$

where contributions to $\mathrm{BS}$ and $\mathrm{BB}$ have been splitted for clarity. In the $\mathrm{Ge}_{2} \mathrm{Sb}_{2} \mathrm{Te}_{5}$ and the $\mathrm{GeSb}_{6}$ systems, one has a total number of constraints which is equal to $n_{c}=3.57$ and 5.26 respectively, i.e. both are highly stressed rigid. The only composition found to be flexible is the $\mathrm{GeTe}_{6}$ composition with $n_{c}=2.73$.

One can now search the Maxwell stability criterion satisfying $n_{c}=D=3$ ( $\mathrm{D}$ being the dimension of the space in which the network is embedded), and corresponding to the mean-field flexible to rigid transition [2],[3]. We furthermore assume that the fraction of tetrahedral units $\eta$ is equal to $x+y$ as it is found that the fraction is 1 for $x+y=1\left(\mathrm{GeSb}_{6}\right)$, and decreases with increasing Te content. With an average coordination number of tellurium of $r_{T e}=2.6$, one finally obtains a constraint map in the Ge-Sb-Te phase diagram (Fig. 3) with a rigidity transition close to the compositional join $\mathrm{GeTe}_{4}-\mathrm{SbTe}_{4}$. 
These results show a profound difference with those obtained in lighter chalcogenides such as Se and S-based networks where usually the isocoordination rule holds [16], i.e. $n_{c}$ does not depend on the considered compositional join as it can be expressed in terms of the network coordination number only (equ. 1) or the cross-link density, which is, by definition, equal to $\left\langle c_{l}\right\rangle=\bar{r}-2$ (Fig. 4).

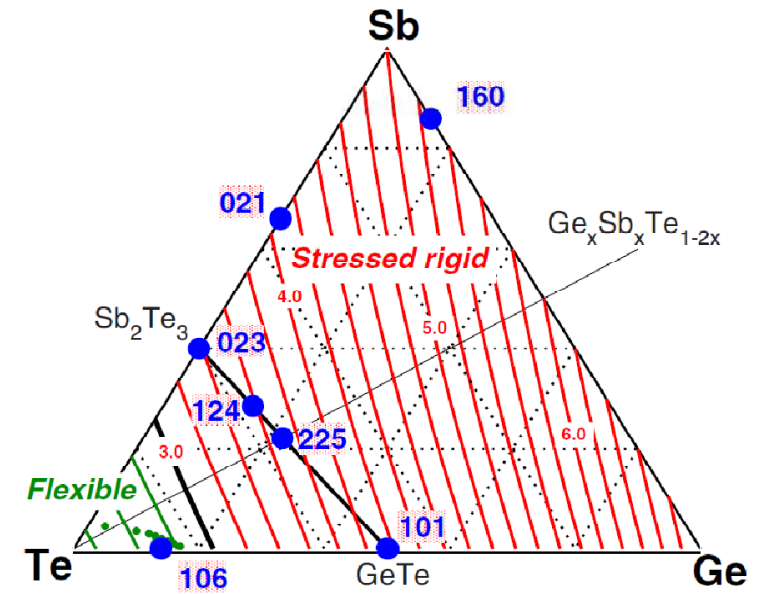

FIGURE 3. Contour map of the number of constraints $n_{c}$ in the ternary $\mathrm{Ge}_{x} \mathrm{Sb}_{y} \mathrm{Te}_{1-x-y}$ phase diagram. The red and green lines correspond, respectively, to the stressed rigid and flexible phase. Blue circles represent the compositions studied by FPMD. The thick black line represents the rigidity transition and separates the flexible (Te rich) from the stressed rigid phase where most PCMs can be found, especially on the GeTe- $\mathrm{Sb}_{2} \mathrm{Te}_{3}$ tie line (black line). Small green dots in the flexible phase represent bulk glass compositions obtained experimentally (Ref. [15]).

When plotted as a function of the latter quantity, and taking into account that the $\mathrm{CN}$ of tellurium also contributes to network cross-linking, it can be seen (Fig. 4) that $n_{c}$ is strongly system dependent in tellurides, with compositions scattered around the 8-N rule line (red) and no clear correlations with the increase of the average angular standard deviation $\left\langle\sigma_{\mathrm{i}}\right\rangle$ computed from the distributions contributing to intact constraints.

Taken together, these results underscore the great difficulty in getting a unified picture in tellurides where a topological description for the understanding of compositional changes of physical behavior may well be strongly system and composition dependent. However, for the present Ge-Sb-Te system, it is found that a mean-field rigidity transition takes place close to the $\mathrm{GeTe}_{4}-\mathrm{SbTe}_{4}$ line which represents the boundary between a Te-rich flexible phase and $(\mathrm{Ge}, \mathrm{Sb})$ crosslinked stressed rigid phase where most of the PCMs are found. Interestingly, one of the great challenges should be the detection of a stress-free intermediate phase in such systems, similarly to the welldocumented selenides [1].

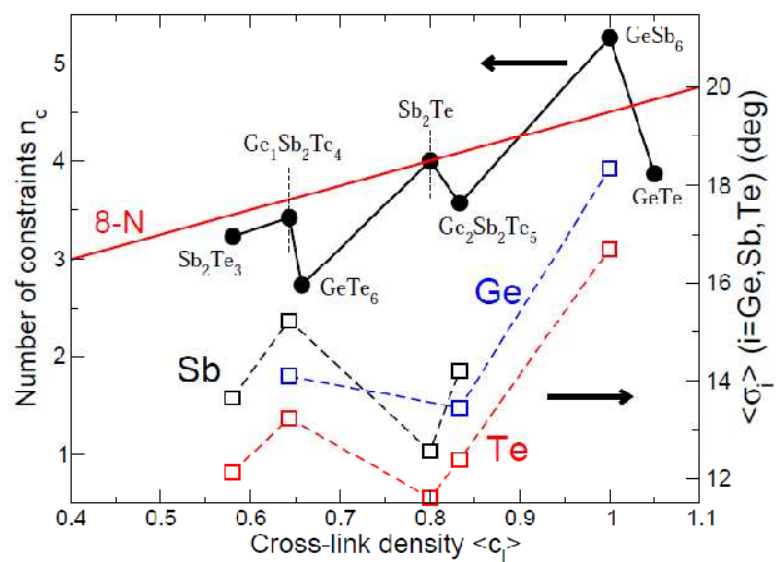

FIGURE 4. Number of constraints as a function of the cross-link density for seven compositions in the ternary $\mathrm{Ge}_{x} \mathrm{Sb}_{y} \mathrm{Te}_{1-x-y}$. compared to what is expected when the 8-N rule holds (red line). Right axis: corresponding $\mathrm{Ge}, \mathrm{Sb}$ and Te mean standard deviation $\left\langle\sigma_{i}\right\rangle$ of the PBAD leading to intact constraints.

\section{REFERENCES}

1. Rigidity and Boolchand Intermediate Phases in Nanomaterials, M. Micoulaut, M. Popescu Eds. INOE Publishing, Bucarest, 2009.

2. J.C. Phillips, J. Non-Cryst. Solids 34, 153 (1979).

3. H. He, M.F. Thorpe, Phys. Rev. Lett. 54, 2107 (1985).

4. Insulating and Semiconducting glasses, P. Boolchand Eds. Singapore, World Scientific, 2000.

5. D. Selvenathan, W. Bresser, P. Boolchand, Phys. Rev. B61, 15061 (2000).

6. S. Chakravarty, D.G. Georgiev, P. Boolchand, M. Micoulaut, J.Phys.Cond.Matt. 17, L7 (2005).

7. F. Wang, S. Mamedov, P. Boolchand, B. Goodman, M. Chandrasekhar, Phys. Rev. B71, 174201 (2005).

8. M. Micoulaut, J.C. Phillips, Phys. Rev. B 67, 104204 (2003).

9. Phase Change Materials: Science And Applications, S. Raoux, M. Wuttig Eds., New York, Springer, 2008.

10. S. Raoux, W. Welnic and D. Ielmini, Chem. Rev. 110, 240-267 (2010)

11. M. Wuttig, Nature Materials, Vol. 4 , p. 265 (2005).

12. D. Lencer, M. Salinga, B. Grabowski, T. Hickel, J. Neugebauer, M. Wuttig, Nature Mat., 7, 972 (2008); M. Wuttig, N. Yamada, Nature Mat., 6, 824 (2007).

13. C. Otjacques, J.-Y. Raty, M.-V. Coulet, M. Johnson, H. Schober, C. Bichara, J.-P. Gaspard, Phys. Rev. Lett. 103, 245901 (2009).

14. M. Micoulaut, C. Otjacques, J-Y. Raty, C. Bichara, Phys. Rev. B 81, 174206 (2010).

15. P. Lebaudy, J. M. Saiter, J. Grenet, M. Belhadji, and C. Vautier, Mater. Sci. Eng., A 132, 273 (1991).

16. B. Effey, R. L. Cappelletti, Phys. Rev. B 59, 4119 (1999). 